\title{
Morbidity and mortality after ileostomy in small bowel perforations
}

\author{
Sumit Raj' ${ }^{1}$ Lonare R. ${ }^{2}$ \\ ${ }^{1}$ Dr. Sumit Raj, Assistant Professor, Department of Nuerosurgery, AIIMS, Bhopal, ${ }^{2}$ Dr. Rajesh Lonare, Associate \\ Professor, Department of Surgery, RKDF Medical College and Research Center, Bhopal, MP, India.
}

Corresponding Author: Dr Rajesh Lonare, Associate Professor, Department of Surgery, RKDF Medical College and Research Center, Bhopal, India. E-mail: dr_raj9@yahoo.com, rajeshlonare09@gmail.com

\begin{abstract}
Background: The aim of the present study was to study the morbidity status of patients of small bowel perforations undergoing ileostomy. To study the intra operative findings determining the construction of type of ileostomy and the effectiveness of various ileostomy appliances in maintaining skin integrity after ileostomy. Methods: The study population consisted of 60 patients of surgically verified ileal perforation in which ileostomy was made. Patients were studied for etiology, site, operative technique, appliance used and complications if any. All the data was presented in the observation tables, analysed and interpretation was done. Results: In our series maximum number of perforations occurred in third decade of life. The youngest patient was 7 years old and oldest patient was 80 year old. Mean age is 29.75 years. The Male: Female ratio is 1.6:1.Abdominal pain was the most prominent symptoms and was found in all patients. Etiologically, typhoid accounts for $63.3 \%$ of all perforation cases, with tuberculosis and traumatic perforation sharing $10 \%$ and $6 \%$ cases each respectively. Out of 60 cases, in which ileostomy was made, 31 patients $(51.6 \%)$ had solitary ileal perforation. Out of 30 patients in whom the Romson's bag was applied, skin excoriation was present in 23 patients (76.6\%). Out of 30 patients in whom the Hollister's bag was applied, only 12 patients (40\%) had skin excoriation. Conclusion: There is a definite reduction in the mortality of the patients of small bowel perforation after ileostomy as compared to primary closure of perforations. Early diagnosis and meticulous surgical interventions of the cause of small bowel perforation and proper post op care is mandatory.
\end{abstract}

Keywords: Morbidity, Mortality, Ileostomy Small Bowel Perforations, Peritonitis

\section{Introduction}

Peritonitis resulting from small bowel perforation is a frequently encountered surgical problem in the tropics. A review of literature indicates a very high mortality associated with this condition in spite of advances in treatment. During the last century advances in antimicrobial therapy, operative techniques, early diagnosis and intensive care environments have produced a profound decrease in mortality from intraabdominal infection.

This improvement reached a plateau with severe intraabdominal infections such as faecal peritonitis, diffuse suppurative peritonitis and necrotising pancreatitis. These entities do not respond to traditional therapies and continue to exhibit high mortality rates [1,2]. The high mortality rate in patients with ileal perforation appears to be a function of the disease process rather

Manuscript Received: $10^{\text {th }}$ July 2019

Reviewed: $20^{\text {th }}$ July 2019

Author Corrected: $29^{\text {th }}$ July 2019

Accepted for Publication: $6^{\text {th }}$ August 2019 than the means of treatment. In favorable circumstances as in strictly localized lesions with a well known etiology and otherwise normal bowel, a simple closure of perforation is warranted. In more advanced cases operated upon early, bowel resection and primary anastomosis is recommended to be the best method of treatment, though it yields poor results in seriously ill patients with most widespread peritonitis. In such patients it is advisable to protect the anastomosis by diverting the faecalsteram by making a controlled fistula [3].

Each type of stoma is associated with a particular spectrum of complications, but some problems are common to all intestinal stomas. The specific ones are dealt with under each category of stoma. A common complication, regardless of the stoma type, is destruction of the peristomal skin, which is usually caused by poor location or construction of the stoma. In addition to the acute maceration and inflammation of 


\section{Research Article}

the skin, pseudoepitheliomatous hyperplasia may arise at the mucocutaneous border of stomas subjected to chronic malfitting appliances. One of the difficult complications to handle, especially in an obese patient, is improper location of the stoma, which prohibits maintenance of the seal of an appliance.

A special problem arises in the patient who has portal hypertension because the construction of a stoma results in the creation of a portosystemic shunt, and varices can form in the peristomal skin [4].

Other common problems include the need for precautions with medications, especially time-released enteric medications, which may pass through a shortened intestinal tract unabsorbed. In some cases the colostomy patient has chronic difficulty maintaining proper fluid and electrolyte balance, and diuretics in these patients can be especially difficult to manage.

The usual intestinal preparations prior to diagnostic testing should be altered for the patient with an intestinal stoma [5].

This study prospectively reviews a series of complications in 60 patients of small bowel perforations in which ileostomy was made.

\section{Materials and Methods}

Setting, duration and type of study- This prospective study was carried out in Department of Surgery in a span of two years.

Patient's Selection- The study population consisted of 60 patients of surgically verified ileal perforation in which ileostomy was made, who were admitted in surgical ward.

Inclusion Criteria-All patients with diagnosed small bowel perforations whether traumatic, inflammatory, mechanical, iatrogenic or spontaneous in which ileostomy was made.

Exclusion Criteria-Patients with perforations of gastric, duodenal, large bowel and appendicular.
Methodology- A written informed consent was taken from all patients. The age, gender, clinical signs and symptoms were recorded. Etiologically the patients were divided into typhoid, tuberculosis and trauma.

Those patients who were diagnosed typhoid on clinical suspicion underwent Widal test. As per history recorded onset of acute pain is regarded as occurring of perforation. Each patient underwent erect abdominal xray examination.

Surgical Procedure-After evaluation, the patient was taken in operation theatre and after opening of abdomen, peritoneal lavage was done and exploration of the gut was done. Intra operative findings were recorded. Then the type of ileostomy was decided as per the intra-operative findings. A suitable section of ileum was chosen and delivered in defect of the abdominal wall. Ileostomy appliance was applied in the postoperative period randomly in 30 patients each. Complications were divided into local and systemic.

Data collection procedure, data analysis-Follow up of patients was done at regular intervals (i.e. within 15 days, 1 month, 3 months and 6 months and the management was done accordingly. In the patients in which there was prolapse or retraction of ileostomy was refashioned. In cases of obstruction and electrolyte imbalance the electrolyte replacement was done and in patients who were not relieved, re-exploration was done. In patients who had burst abdomen the tension suturing of the abdomen was done.

All the data was presented in the observation tables, analysed and interpretation was done. Suitable statistical test were applied for the significance.

Statistical Analysis- The data of the present study were fed into the computer and after its proper validation, checking for error, coding and decoding were compiled and analysed with the help of SPSS 11.5 software for windows. After obtaining the data, the two procedures were compared using student t-test, chi square test and odds ratio. Then the $\mathrm{p}$ value was obtained using Microsoft Excel software and Epical 1.0 software and the statistical significance of the results were compared.

\section{Results}

In our series maximum number of perforations occurred in third decade of life. The youngest patient was 7 years old and oldest patient was 80 year old. Mean age is 29.75 years. The Male:Female ratio is $1.6: 1$. Abdominal pain was the most prominent symptoms and was found in all the 60 patients (100\%). Majority of them had a history of vomiting (83\%), fever was seen in 46 patients $(76.6 \%)$. In patients with enteric etiology, 30 out of 46 patients were having fever of average duration of 15 days. 


\section{Research Article}

Patients having tuberculous etiology had history of long duration of fever. Diarrhoea was uncommon presentation as most of the patients were in paralytic ileas. Constipation and distension of abdomen was seen in 50 patients (86.6\%) and 52 patients $(83.3 \%)$ respectively. Tachycardia was the most common sign and was present in 52 patients $(86.6 \%)$. Tachypnoea was present in 28 patients $(66.6 \%)$ who had long duration of perforation. Oligouria was present in only 6 patients $(10 \%)$.

Etiologically, typhoid accounts for $63.3 \%$ of all perforation cases, with tuberculosis and traumatic perforation sharing $10 \%$ and $6 \%$ cases each respectively. In 12 patients $(20 \%)$ the etiology of the perforations were non-specific. 44 patients (73.3\%) were operated within 72 hours of perforation. Most of the patients $(63.3 \%)$ presented to the hospital within 37 to 48 hours of perforation. Pneumoperitoneum was seen in 38 out of 60 patients $(63.3 \%)$, in home abdominal X-ray was taken. In 14 patients (23.3\%), erect abdominal x-ray film showed multiple air-fluid levels suggestive of intestinal obstruction. Out of 60 patients, 8 patients (13.3\%) showed no significant finding in erect abdominal X-ray films.

Out of 60 cases, in which ileostomy was made, 31 patients $(51.6 \%)$ had solitary ileal perforation. The number of perforations were two in 14 patients $(23.3 \%)$. More than two perforations were found in 15 patients $(25 \%)$.

In 29 patients the perforation were located within $30 \mathrm{cms}$ from ileocecal junction $(48.3 \%)$. In 20 patients $(33.3 \%)$, the perforation was located within $30-60 \mathrm{cms}$ from the ileocecal junction. In 11 patients $(18.3 \%)$, the perforation was located more than $60 \mathrm{cms}$ from the ileocecal junction.

Table-1: Clinical symptoms of patients.

\begin{tabular}{|c|c|c|c|}
\hline S.No. & Symptoms & No. & \% \\
\hline 1. & Pain in abdomen & 60 & 83.33 \\
\hline 2. & Vomiting & 50 & 76.66 \\
\hline 3. & Fever & 46 & 3.33 \\
\hline 4. & Diarrhoea & 52 & 86.66 \\
\hline 5. & Distention & 50 & 83.33 \\
\hline 6. & Constipation & 4 & 6.66 \\
\hline 7. & H/o Trauma & & 2 \\
\hline
\end{tabular}

Table-2: Clinical signs of patients.

\begin{tabular}{|c|c|c|c|}
\hline S.No. & Signs & No. & \% \\
\hline 1. & Tachycardia $(>100 / \mathrm{min})$ & 52 & 86.66 \\
\hline 2. & Hypotension $(<100 \mathrm{mmHg}$ systolic $)$ & 40 & 46.66 \\
\hline 3. & Tachypnoea $(>20 / \mathrm{min})$ & 28 & 10 \\
\hline 4. & Urine output $(<30 \mathrm{ml} / \mathrm{hr})$ & 6 & 46.66 \\
\hline
\end{tabular}

Table-3: Etiology Of Patients.

\begin{tabular}{|c|c|c|c|}
\hline S.No. & Etiology & No. & \% \\
\hline 1. & Typhoid & 38 & 63.33 \\
\hline 2. & Tubercular & 6 & 6.67 \\
\hline 3. & Traumatic & 12 & 20 \\
\hline 4. & Non specific & $\mathbf{6 0}$ & $\mathbf{1 0 0}$ \\
\hline
\end{tabular}


Research Article

Table-4: Site of perforation.

\begin{tabular}{|c|c|c|c|}
\hline S.No. & Site of perforation from ileo-caecal junction & No. of cases & $\%$ \\
\hline 1. & Less than $30 \mathrm{~cm}$ & 29 & 48.33 \\
\hline 2. & $30-60 \mathrm{~cm}$ & 20 & 33.33 \\
\hline 3. & More than $60 \mathrm{~cm}$ & 11 & 18.33 \\
\hline & Total & $\mathbf{6 0}$ & $\mathbf{1 0 0}$ \\
\hline
\end{tabular}

Table-5: Type of ileostomy performed according to the intraoperative findings.

\begin{tabular}{|c|c|c|c|c|c|c|}
\hline \multirow{2}{*}{$\begin{array}{l}\text { S. } \\
\text { No. }\end{array}$} & \multirow{2}{*}{$\begin{array}{c}\text { No. of } \\
\text { perforation }\end{array}$} & \multirow{2}{*}{$\begin{array}{c}\text { Site of } \\
\text { perforation }\end{array}$} & \multicolumn{4}{|c|}{ Operative procedure } \\
\hline & & & $\begin{array}{l}\text { Exteriorisation } \\
\text { of perforation }\end{array}$ & $\begin{array}{c}\text { Distal repair } \\
\text { with proximal } \\
\text { ileostomy }\end{array}$ & $\begin{array}{c}\text { Double } \\
\text { barrel } \\
\text { ileostomy }\end{array}$ & $\begin{array}{l}\text { End } \\
\text { ileostomy }\end{array}$ \\
\hline \multirow[t]{3}{*}{1.} & \multirow[t]{3}{*}{ One } & A. $<30 \mathrm{~cm}$. & Nil & 13 & Nil & 1 \\
\hline & & B. $30-60 \mathrm{~cm}$. & 10 & Nil & Nil & Nil \\
\hline & & $\mathrm{C}>60 \mathrm{~cm}$ & 7 & Nil & Nil & Nil \\
\hline \multirow[t]{3}{*}{2.} & \multirow[t]{3}{*}{ Two } & A. $<30 \mathrm{~cm}$. & 1 & 8 & Nil & 4 \\
\hline & & B. $30-60 \mathrm{~cm}$. & Nil & Nil & Nil & Nil \\
\hline & & $\mathrm{C}>60 \mathrm{~cm}$. & 2 & Nil & Nil & Nil \\
\hline \multirow[t]{3}{*}{3.} & \multirow{3}{*}{$\begin{array}{l}\text { More than } \\
\text { two }\end{array}$} & A. $<30 \mathrm{~cm}$. & Nil & Nil & Nil & 3 \\
\hline & & B. $30-60 \mathrm{~cm}$. & Nil & 1 & 7 & 1 \\
\hline & & $\mathrm{C}>60 \mathrm{~cm}$ & Nil & Nil & 1 & 1 \\
\hline & \multicolumn{2}{|l|}{ Total } & 20 & 22 & 8 & 10 \\
\hline
\end{tabular}

Table-6: Local postoperative complications.

\begin{tabular}{|c|c|c|c|}
\hline S.No. & Complication & No. of cases & \% \\
\hline 1. & Bleeding & 12 & 6.66 \\
\hline 2. & Necrosis & 7 & 11.66 \\
\hline 3. & Stenosis & 2 & 3.33 \\
\hline 4. & Parastomal hernia & 34 & 56.66 \\
\hline 5. & Skin excoriation & 9 & 15 \\
\hline 6. & Retraction & 5 & 8.33 \\
\hline 7. & Prolapse & 14 & 23.33 \\
\hline 8. & Obstruction & 32 & 53.33 \\
\hline 9. & Midline wound dehiscence & 14 & 23.33 \\
\hline 10. & Burst abdomen & 43 \\
\hline
\end{tabular}


Research Article

Table-7: Systemic Postoperative Complications.

\begin{tabular}{|c|c|c|c|}
\hline S.No. & Complication & No. of cases & \% \\
\hline 1. & Electrolyte imbalance & 32 & 53.33 \\
\hline 2. & Pulmonary Infection & 23 & 38.33 \\
\hline 3. & Renal Failure & 15 & 25 \\
\hline 4. & Encephalopathy & 14 & 23.33 \\
\hline 5. & Septicemia & 8 & 13.33 \\
\hline
\end{tabular}

Out of all procedures performed, the repair of distal perforation with proximal loop ileostomy and exteriorization of solitary ileal perforation were more common i.e., 21 (35\%) and $22(36.6 \%)$ respectively. Exteriorization of ileal perforation was performed commonly in which patients had solitary perforation with distance $30-60 \mathrm{cms}$. or more than $60 \mathrm{cms}$. for ileocaecal junction. Repair of distal perforation with proximal loop ileostomy was performed in patients with one or two perforations with distance less than $30 \mathrm{cms}$. from the ileocaecal junction. Resection of perforated ileal segment with double barrel ileostomy was performed commonly in patients with more than two perforations and distance 30 $60 \mathrm{cms}$ or more than $60 \mathrm{cms}$ from ileocaecal junction. End ileostomy was performed commonly in patients with two or more than two perforations and distance less than $30 \mathrm{cms}$ for the ileocaecal junctions kin excoriation was present in 35 patients $(58.3 \%)$ out of 60 . Out of 30 patients in whom the Romson's bag was applied, skin excoriation was present in 23 patients $(76.6 \%)$. Out of 30 patients in whom the Hollister's bag was applied, only 12 patients (40\%) had skin excoriation.

Midline wound dehiscence and skin excoriation were the most common local complication. Parastomal hernia was seen in only 2 patients $(3.3 \%) .14$ patients $(23.3 \%)$ out of 60 , presented with burst abdomen. Electrolyte imbalance and pulmonary infection were the systemic complication seen in most of the patients. Out of 60 patients, all the patients having septicemia were expired. As per the collected data, rest of the complications were also associated with the expired cases. Duration of stay after the operation was less than 15 days in 15 patients $(25 \%)$, 15 days to 1 month in 36 patients and more than 1 month stay in 9 patients (15\%). Out of 60 patients, 52 were recovered and 8 patients died inspite of all resuscitative measures.

\section{Discussion}

The present study consisted of 60 patients of surgically verified ileal perforation in which ileostomy was made, who were admitted in surgical ward our hospital over a span of 3 years. Small bowel perforation most commonly affects young men in the prime of life. Typhoid perforation is rare under the age of 5 years and over the age of 50 years. Typhoid perforation usually occurs in the third week of fever In present study maximum incidence was found in the third week. The reason for this was because of inadequate and symptomatic treatment by the registered medical practitioners and quacks which caused relief of symptoms initially but later on leads to the perforation and other complications in enteric fever.

O'connor A, Sawin RS et al studied morbidity of enterostomy and its closure in premature infants with necrotizing enterocolitis. A group of 68 infants aged 2 to 35 days (mean age, 12.5 days), weighing $1500 \mathrm{~g}$ or less, with necrotizing enterocolitis necessitating surgical enterostomy for treatment. They concluded that although enterostomy in infants with low birth weight with necrotizing enterocolitis may be lifesaving, it is also a major cause of morbidity [1]. Chow A et al did a systematic review on the the morbidity surrounding reversal of defunctioning ileostomies. The aims of this systematic review were to examine all the existing evidence in the literature on morbidity and mortality following closure of loop ileostomy.Forty-eight studies from 18 countries satisfied the inclusion criteria. Outcomes of a total of 6,107 patients were analysed. Overall morbidity following closure of loop ileostomy was found to be $17.3 \%$ with a mortality rate of $0.4 \%$. $3.7 \%$ of patients required a laparotomy at the time of ileostomy closure.

The most common post-operative complications included small bowel obstruction (7.2\%) and wound sepsis $(5.0 \%)$. They concluded that the consequences of anastomotic leakage following colorectal resection are severe. However, the consequences of stoma reversal are often underestimated. Surgeons should adopt a 


\section{Research Article}

selective strategy regarding the use of defunctioning ileostomy, and counsel patients further prior to the original surgery. In this way, patients at low risk may be spared the morbidity of stoma reversal [2].

Gupta PK et al studied Morbidity and mortality after bowel resection for acute mesenteric ischemia. Patients presenting with acute mesenteric ischemia (AMI) sufficiently advanced to require bowel resection have a high morbidity and mortality. The objective of this study was to analyze these patients to determine if certain pre- or intraoperative variables are predictive of death or complications which could then be used to develop a predictive model to aid in surgical decisionmaking. The study concluded that mortality and morbidity rates after bowel resection for AMI are high. A risk calculator for prediction of postoperative mortality and morbidity has been developed and awaits validation in subsequent studies [3].

The creation of a loop ileostomy is considered suitable to protect a distal anastomosis in colorectal surgery. This technique is, however, associated with failure, complications and even mortality. The aim of study done by Giannakopoulos GF et al was to quantify retrospectively the morbidity associated with an ileostomy and its subsequent closure. Protective loop ileostomy was found to be associated with a high morbidity. This raises the question of the mode of identifying the specific patients with a low anastomosis who should be provided an ileostomy for protection, set against the potential complications of the formation and closure of the ileostomy [4].

Harberg FJ et al explored the possibilities of resection with primary anastomosis for necrotizing enterocolitis. Primary anastomosis following the resection of bowel for necrotizing enterocolitis (NEC) can be done with acceptable morbidity and mortality rates. Twenty-seven patients are reported with three deaths. Primary anastomosis will avoid problems seen with small-bowel stomas. The concomitant ligation of a significant patent ductus arteriosus (PDA) should be considered for patients undergoing abdominal procedures for NEC[5].

Prognostic factors for survival in colonic perforation were studied by Kriwanek $\mathrm{S}$ et al. He found that Colonic perforation is an abdominal emergency with high morbidity and mortality. This retrospective study was performed to evaluate the prognostic relevance of several factors and to characterize patients at high risk. The overall mortality was $19.6 \%$ (22 patients). The prognostic importance of various factors was investigated by univariate analysis (Wilcoxon and Chisquare test) and stepwise logistic regression including sex, age, underlying disease, localization and type of perforation, degree of peritonits, per-or postoperative organ failure, surgical procedure, reoperation, sepsis and the Mannheimer Peritonitis Index (MPI) score. Age over 65 years (relative risk 4.6, $\mathrm{P}=0.0089$ ), organ failure (relative risk $40, \mathrm{P}=0.001$ ) and MPI (relative risk for an increase of 10 points $2.72, \mathrm{P}=0.001$ ) proved to be the only risk factors of significance. The patient's course is determined by the septic state, while the underlying pathology and degree of peritonitis did not significantly influence survival. The results of the study are very similar to the present study [6].

Nadkarni KM et al did a study of 32 cases of smallbowel perforations. 32 consecutive cases of smallbowel perforations treated in a single surgical unit during the last three years. Clinical features are typical and diagnosis is not difficult. Suprapubic peritoneal paracentesis in head-high position is the most accurate diagnostic investigation. Ultimate results are not related to cause, but are directly proportional to the degree of contamination of the peritoneal cavity, delay in manifestation, antibiotic resistance of the contaminating organism, and the method of treatment of the perforation. There were two deaths in the 16 patients who had exteriorization of the suture line (12.5\%), compared with seven deaths in the group of 16 patients who did not have exteriorization (43.75\%). Exteriorization of the suture line (16 cases) is a superior method of treatment and significantly lowers the mortality [7].

Eggleston FC et al shared their experience of 78 cases of typhoid perforation of the bowel. Patients ranging from four to 65 years of age were treated for typhoid perforation of the bowel. Sixty-one patients (78\%) were males. The average time from perforation to admission was 56 hours. The mortality rate was $32 \%$ and was adversely influenced by the duration of illness, duration of perforation, shock, uremia, encephalopathy and fecal peritonitis. Forty-nine patients were treated by closure of the perforation, resection or miscellaneous procedures; the other 29 by closure of the perforation combined with an end-to-end ileotransverse colostomy. Although mortality was the same in both groups, those undergoing bypass had a significantly smoother postoperative course [8]. Singh KP et al in a similar study shared their experience in 42 cases and made their choice of surgical procedure in typhoid perforation. All the patients were subjected to surgery after resuscitation. Simple closure (with or without serosal 


\section{Research Article}

patch) and temporary ileostomy were the commonly performed surgical procedures. The overall mortality was $14.2 \%$. Postoperative morbidity included wound dehiscence, fistula, encephalopathy, septicaemia, intraabdominal abscesses and incisional hernia. Faecal fistula formed in 5 out of 42 cases. Ileostomy was found to be the best procedure with regard to morbidity and mortality [9].

Eustache JM et reviewed 91 cases of intestinal perforation complicating typhoid fever treated at a rural hospital in Haiti over a ten-year period. Surgical management involved simple primary closure of the perforation (80 patients), small-bowel resection with anastomosis (two patients), simple drainage of the peritoneal cavity (two patients), and serosal patching of the perforation (one patient). Six patients died before surgery. The mortality was $30.8 \%$ for all 91 cases but $21.2 \%$ for those treated with primary closure of the perforation. They also reviewed the literature pertaining to the management of intestinal perforation complicating typhoid fever[10].

Khanna AK etal did their study on typhoid perforation of the gut .One-hundred consecutive patients with typhoid perforation of the gut admitted in the same surgical unit of the University Hospital have been studied. The cases were diagnosed on the basis of history, clinical examination, exploratory findings, histopathological examination, Widal test and blood culture. Forty-six patients had perforation in the second week of fever. Sixty-one patients presented 48-96 hr after perforation. All the patients were subjected to surgery, 16 under local anesthesia. Mortality rate increased from $25 \%$ to $83 \%$ as the duration between perforation and operation increased [11].

Problems in the management of abdominal tuberculosis are discussed with reference to 300 surgically verified cases by Bhansali SK et al. The protean clinical manifestations depends on the site and extent of the disease, and its complications. Operation was resorted to for complications when diagnosis was in doubt and when intrinsic intestinal disease was proved. Surgery was preceded by antituberculous drugs whenever possible. At operation, the disease was found to involve the alimentary canal in 196 cases; in the remaining 104, only the lymph nodes and/or the peritoneum were affected. Intestinal resection was carried out in 100 cases. Emergency surgery carries a high mortality $(18 / 76)$ because of toxemia, hypoproteinemia, anemia, etc. Positive histology was obtained in 229 cases. One hundred and seventy-nine cases showed evidence of caseation. Caseation and peritoneal tubercles (103 cases) differentiate intestinal tuberculosis from Crohn's disease. Despite considerable progress made in therapy and prophylaxis during the last quarter of the century, tuberculosis of various sites continues to be a major health hazard in India. The precise prevalence of Koch's disease of the abdomen has not been determined due to lack of a survey in random samples of population. This common malady, however, with its protean profiles and varied complications continues to challenge the diagnostic acumen and therapeutic skill of clinicians practicing various discplines of medicine[12].

Malik AM et al explored different surgical options and ileostomy in typhoid perforation to find out the value of primary ileostomy as a life saving procedure in patients of typhoid ileal perforation. 112 diagnosed cases were included in this study with a mean age of 18.66 years with a male to female ratio of 1.5: 1. Talwar $\mathrm{S}$ et al also did a similar study on Typhoid enteric perforation. The overall mortality rate was $16.4 \%$. Increasing the time interval between perforation and operation significantly increased the mortality $(\mathrm{P}<0.05)$. The mortality was least with early primary closure of the perforation. Patients with postoperative faecal fistula had higher mortality rates $(\mathrm{P}<0.001)$.It was concluded that early limited surgery with thorough peritoneal lavage provides optimal results, faecal fistula is a grave complication, and the use of the Mc Burney incision may provide better results in terms of subsequent wound healing $[13,14]$.

Leong AP et al did a life-table analysis of stomal complications following ileostomy. Stomal complications of ileostomy may occur many years after construction. An actuarial analysis of complications of 150 permanent end ileostomies constructed over a 10 -year period is reported. By 20 years the incidence of stomal complications approached 76 per cent in patients operated on for ulcerative colitis and 59 per cent in those with Crohn's disease $(\mathrm{P}<0.05)$. Revisional surgery rates were higher in patients with ulcerative colitis than in those with Crohn's disease (28 versus 16 per cent), albeit not significantly.

The four commonest complications were skin problems (cumulative probability 34 per cent), intestinal obstruction ( 23 per cent), retraction (17 per cent) and parastomal herniation (16 per cent). Closure of the lateral space did not reduce the probability of developing intestinal obstruction (18 per cent at 20 years in those with closure versus 3 per cent in those without, Pgt; $0 \cdot 1)$. Fixation of the mesentery did not 


\section{Research Article}

reduce the probability of developing prolapse of the ileostomy (11 per cent in those with fixation versus none in those without, $\mathrm{P}<0 \cdot 1)$. The incidence of parastomal herniation was not reduced by siting through the rectus abdominis ( 21 per cent in those sited through the body of the rectus abdominis versus 7 per cent in those sited through the oblique muscles, $\mathrm{P}<0 \cdot 1$ ). Some of the surgical dogmas relating to ileostomy construction are not supported by the results of this study $[15,16]$.

In the present study, it was found that out of 30 patients in which Hollister's bag was applied skin excoriation was found to be present in 12 cases (40\%) while 23 patients $(76.6 \%)$ using Romson's bag had skin excoriation. Pearson's Chi - square test was applied and the $\mathrm{p}$ value was found to be $<0.05 \%$ i.e., highly significant. Hence Hollister's bag is recommended as it reduces the incidence of skin excoriation around the stoma.

Mortality rates from other studies are comparable to the present study i.e., $13.3 \%$. Hence, the primary ileostomy is a better option in cases of ileal perforation. In the present study the overall $13.3 \%$ mortality in ileal perforations treated by ileostomy, as compared with other studies who had shown a high mortality in their ileal perforations who were treated with primary closure. Therefore it is recommend that early ileostomy reduces mortality.

\section{Limitation of the present study}

1. Small sample size

2. Chances of bias

3. Single center trial

\section{Conclusion}

- The increase in the operation - perforation interval increases the morbidity and mortality of the patients of ileal perforation. Skin excoriation is found to be much less in Hollister bag's application as compared to Romson's bag in ileostomy.

- There is a definite reduction in the mortality of the patients of small bowel perforation after ileostomy as compared to primary closure of perforations.

- However the morbidity rate following the ileostomy is not much affected but early diagnosis and meticulous surgical interventions of the cause of small bowel perforation and proper post op care is mandatory.
However, these conclusions are drawn by a study of 60 patients which is a small group.

So a larger group of patients in the study were required to come to a statistical significant conclusion.

\section{What this study adds to existing knowledge?}

The primary ileostomy is a better option in cases of ileal perforation.

In the present study the overall $13.3 \%$ mortality in ileal perforations treated by ileostomy, as compared with other studies who had shown a high mortality in their ileal perforations who were treated with primary closure.

\section{Author's contribution}

Dr. Sumit Raj: Study design, data analysis and manuscript preparation.

Dr. Rajesh Lonare: Study design, data analysis and manuscript preparation.

Conflict of interest: None declared.

Funding: Nil, Permission from IRB: Yes

Ethical approval: Taken

\section{References}

1. O'Connor A, Sawin RS. High morbidity of enterostomy and its closure in premature infants with necrotizing enterocolitis. Archives of Surgery. 1998; 133 (8):875-80.

2. Chow A, Tilney HS, Paraskeva P, Jeyarajah S, ZacharakisE, Purkayastha S. The morbidity surrounding reversal of defunctioning ileostomies: a systematic review of 48 studies including 6,107 cases. Int $\mathrm{J}$ Colorectal Dis. 2009; 24(6):711-23. doi: 10.1007/ s 00384-009-0660-z. Epub 2009.

3. Gupta PK, Natarajan B, Gupta H, Fang X, Fitzgibbons RJ Jr. Morbidity and mortality after bowel resection for acute mesenteric ischemia. Surg. 2011; 150 (4):779-87. doi: 10.1016/j.surg. 2011. 07.079.

4. Giannakopoulos GF, Veenhof AA, van der Peet DL, Sietses C, Meijerink WJ, Cuesta MA. Morbidity and complications of protective loop ileostomy. Colorectal Dis. 2009;11(6):609-12. doi:10.1111/j.1463-1318.2008. 01690.x. Epub 2008. 


\section{Research Article}

5. Harberg FJ, McGill CW, Saleem MM, Halbert $\mathrm{R}$, Anastassiou P. Resection with primary anastomosis for necrotizing enterocolitis. J Pediatr Surg. 1983;18 (6): 743-6. doi:10.1016/s0022-3468(83) 80016-5

6. Kriwanek S, Armbruster C, Dittrich K, Beckerhinn P. Perforated colorectal cancer. Dis Colon Rectum. 1996; 39 (12): 1409-14. doi:10.1007/bf02054530

7. Nadkarni KM, Shetty SD, Kagzi RS, Pinto AC, Bhalerao RA. Small-bowel perforations. A study of 32 cases. Arch Surg. 1981;116(1):53-7. doi:10.1001/ archsurg.1981.01380130033008

8. Eggleston FC, Santoshi B, Singh CM. Typhoid perforation of the bowel. Experiences in 78 cases. Ann Surg. 1979;190(1):31-5.doi:10.1097/00000658-19790 $7000-00007$

9. Singh KP, Singh K, Kohli JS. Choice of surgical procedure in typhoid perforation: experience in 42 cases. J Indian Med Associat. 1991;89(9):255-6.

10. Eustache JM, Kreis DJ Jr. Typhoid perforation of the intestine. Arch Surg. 1983;118(11):1269-71. doi:10. 1001 / archsurg.1983.01390110027007
11. Khanna AK, Misra MK. Typhoid perforation of the gut. Postgrad Med J. 1984;60(706):523-5. doi:10. 1136/ pgmj.60.706.523

12. Bhansali SK. Abdominal tuberculosis. Experiences with 300 cases. Am J Gastroenterol. 1977 Apr;67 (4): 324-37.

13. Malik AM, Laghari AA, Mallah Q, Qureshi GA, Talpur AH, Effendi S, Memon JM. Different surgical options and ileostomy in typhoid perforation. World $\mathrm{J}$ Med Sci. 2006;1(2):112-6.

14. Talwar S, Sharma RK, Mittal DK, Prasad P. Typhoid enteric perforation. Aust NZ J Surg. 1997;67 (6):351-3. doi:10.1111/j.1445-2197.1997.tb01990.x

15. Leong AP, Londono-Schimmer EE, Phillips RK. Life-table analysis of stomal complications following ileostomy. Br J Surg. 1994 ;81(5):727-9. doi:10.1002/ bjs. 1800810536

16. Olurin EO, Ajayi OO, Bohrer SP. Typhoid perforations. J R Coll Surg Edinb. 1972 ;17(6):353-63.

\section{How to cite this article?}

Sumit Raj, Lonare R. Morbidity and mortality after ileostomy in small bowel perforations. Surgical Update: Int J surg Orthopedics. 2019;5(3):167-175. doi:10.17511/ijoso.2019.i03.07. 